\title{
Aplicação da toxina botulínica tipo A como alternativa para a correção do sorriso gengival: Uma revisão de literatura
}

\author{
Type A botulinum toxin application as an alternative for gingival smile correction: A literature \\ review
}

Escriba una aplicación de botulinum toxin como alternativa para la corrección de sonrisa gingival: Una revisión de la literatura

\section{Resumo}

A busca pela excelência estética é uma das metas da odontologia, pois faz parte da estética do sorriso, pois interfere diretamente na autoestima e na qualidade de vida do paciente. Conceitualmente, o sorriso gengival é a exposição excessiva de gengiva ao sorrir, podendo ser considerada a partir de $2 \mathrm{~mm}$. Uma das opções de correção é com o uso da toxina botulínica. Em vista disso, o objetivo do presente estudo foi realizar uma revisão da literatura sobre os estudos científicos atuais acerca da correção do sorriso gengival com a toxina botulínica tipo A. Para tanto, realizou-se uma busca dos principais artigos publicados nos últimos 5 anos nas bases de dados: PubMed, SciELO, LILACS e BVS utilizando como descritores inglês "smile", "gummy smile", "gingiva" e "Botulinum ToxinsType A". Baseado no resultado da busca dos 22 artigos encontrados, o tratamento do sorriso gengival com toxina botulínica tipo A atua sobre a hiperatividade dos músculos envolvidos no sorriso causando um relaxamento temporário, podendo ser uma técnica independente ou coadjuvante de outros procedimentos. Apresentando eficácia, resultados positivos estéticos e baixo índice de complicações. No entanto, a principal desvantagem consiste no efeito temporário, com duração média de 4 a 6 meses, necessitando de reaplicações com o passar do tempo. Diante desse contexto, pode-se concluir que o uso desta toxina para a redução do sorriso gengival é uma opção de procedimento seguro, rápido, eficaz e conservador. Entretanto, ensaios clínicos randomizados bemdelineados são necessários para fortalecer as evidências.

Palavras-chave: Sorriso; Gengiva; Toxinas botulínicas tipo A.

\begin{abstract}
The search for aesthetic excellence is one of the goals of dentistry, as it is part of the aesthetics of the smile, as it directly interferes with the patient's self-esteem and quality of life. Conceptually, the gingival smile is the excessive exposure of the gingiva when smiling, and can be considered from $2 \mathrm{~mm}$. One of the correction options is with the use of botulinum toxin. In view of this, the aim of this study was to conduct a literature review on current scientific studies on the correction of gummy smile with botulinum toxin type A. Therefore, a search of the main articles published in the last 5 years in the databases: PubMed, SciELO, LILACS and VHL using English as descriptors "smile", "gummy smile", "gingiva" and "Botulinum ToxinsType A". Based on the search result of the 22 articles found, the treatment of gummy smile with botulinum toxin type A acts on the hyperactivity of the muscles involved in the smile, causing a temporary relaxation, which may be an independent technique or an adjunct to other procedures. Showing efficacy, positive esthetic results and low complication rate. However, the main disadvantage is the temporary effect, with an average duration of 4 to 6 months, requiring reapplications over time. Given this context, it can be concluded that the use of this toxin to reduce gummy smile is a safe, fast, effective and conservative procedure option. However, well-designed randomized controlled trials are needed to strengthen the evidence.
\end{abstract}

Keywords: Smile; Gum; Botulinum toxins type A.

\section{Resumen}

La búsqueda de la excelencia estética es uno de los objetivos de la odontología, ya que forma parte de la estética de la sonrisa, ya que interfiere directamente en la autoestima y calidad de vida del paciente. Conceptualmente, la sonrisa gingival es la exposición excesiva de la encía al sonreír, y se puede considerar a partir de $2 \mathrm{~mm}$. Una de las opciones 
de corrección es con el uso de toxina botulínica. Ante esto, el objetivo del presente estudio fue realizar una revisión de la literatura sobre los estudios científicos actuales sobre la corrección de la sonrisa gingival con toxina botulínica tipo A. Por tanto, se realizó una búsqueda de los principales artículos publicados en los últimos 5 años en el Bases de datos: PubMed, SciELO, LILACS y VHL utilizando el inglés como descriptores "smile", "gummy smile", "gingiva" y "Botulinum ToxinsType A". Con base en el resultado de la búsqueda de los 22 artículos encontrados, el tratamiento de la sonrisa gingival con toxina botulínica tipo A actúa sobre la hiperactividad de los músculos involucrados en la sonrisa, provocando una relajación temporal, que puede ser una técnica independiente o coadyuvante a otros procedimientos. Demostrando eficacia, resultados estéticos positivos y baja tasa de complicaciones. Sin embargo, la principal desventaja es el efecto temporal, con una duración promedio de 4 a 6 meses, requiriendo reaplicaciones a lo largo del tiempo. En este contexto, se puede concluir que el uso de esta toxina para reducir la sonrisa gingival es una opción de procedimiento segura, rápida, eficaz y conservadora. Sin embargo, se necesitan ensayos controlados aleatorios bien diseñados para fortalecer la evidencia.

Palabras clave: Sonrisa. Encía. Toxinas botulínicas tipo A.

\section{Introdução}

Atualmente, o conceito de parâmetros e padrões estéticos na Odontologia, assim como a satisfação do paciente vem sendo bastante discutido por cirurgiões-dentistas e se tornando um dos princípios de promoção de saúde (Araújo, Cruz, Oliveira \& Canto, 2018). Em contrapartida, a definição de beleza é subjetiva,dependendo de cada indivíduo, que sofre influências por variáveis psicológica, sociocultural e econômica as quais devem ser respeitadas e analisadas dentro da ética e dos limites do procedimento pelo profissional (Gupta \& Kohli, 2019).

Nesse contexto, o sorriso exerce importante papel na estética facial influenciando de forma direta na autoestima e consequentemente na qualidade de vida do paciente. Assim, a odontologia estética requer bastante atenção na hora de identificar problemas que estejam causando desconforto e tratar cada paciente individualmente, respeitando as limitações do mesmo e as necessidades de cada um, assim o diagnóstico e o planejamento se tornam essenciais (Araújo, Cruz, Oliveira \& Canto, 2018).

Dessa maneira, um dos fatores que podem impactar na percepçãoestética, é o sorriso gengival, que é definido com uma exposição excessiva de gengiva durante o sorriso, que pode variar entre diferentes indivíduos. O mesmo tem sido motivo de desconforto para muitos pacientes nos últimos anos, o que está relacionado ao aumento da procura nos consultórios para a correção desta condição (Rego, Santos \& Pedron, 2015).

Para tanto se faz necessário o diagnóstico, pois o sorriso gengival apresenta diferentes etiologias, podendo está relacionado com o formato dental, as características do contorno gengival, o posicionamento labial e a hiperatividade dos músculos envolvidos no sorriso como causa. Dessa forma, podendo ter mais de uma alternativa de escolha para a correção ou a combinação de duas ou mais técnicas (Brito, Alves, Sousa, Rodrigues \& Souza, 2016).

Por muito tempo, a correção cirúrgica era a única forma de tratamento mesmosendo invasiva e com custo não tão acessível. Porém, o uso da toxina botulínica tipo A, conhecida comercialmente como botox, surge como alternativa não invasiva, mais acessível financeiramente, reversível e com resultados satisfatórios como forma de tratamento (Moreira, Possidônio, Souza, Kinoshita \& Silveira, 2019).

A toxina botulínica tipo A é uma toxina produzida pela fermentação de uma bactéria anaeróbica, sendo segura sua aplicação por um cirurgião dentista ou médico capacitado e especializado. Diante disso, existem recomendações, mecanismo BTXsucesso da técnica (Rego, Santos \& Pedron, 2015).

Diante dessas considerações, o objetivo do presente estudo foi revisar aliteratura sobre as evidências científicas atuais acerca do uso da toxina botulínica tipo A como alternativa para a correção do sorriso gengival. 


\section{Metodologia}

O presente trabalho trata-se de uma revisão da literatura recente sobre correção do sorriso gengival com toxina botulínica tipo A. Os artigos utilizados neste estudo foram selecionados por meio de busca online nas bases de dados: Sistema Online de Busca e Analise da Literatura Médica- MEDLINE (via PubMed), Literatura Latino-Americana e do Caribe em Ciências da Saúde- LILACS (via Biblioteca Nacional de Saúde (BVS) e Biblioteca Eletrônica Científica Online- SciELO.

Na pesquisa dos artigos foram utilizados os descritores inglês "smile", "gummy smile", "gingiva" e "Botulinum ToxinsType A". Além disso, para a leitura e seleção dos artigos foi criado um acervo no gerenciador de referências Mendeley Desktop (Version 1.16.1, Mendeley Ltd., Elsevier Inc., NY, USA).

Foram objetos de análise exploratória artigos de revisão de literatura, estudos de coorte, revisão sistemática e casos clínicos. Os artigos foram selecionados de acordo com sua relevância, através da leitura dos resumos e análise detalhada dos mesmos de acordo com os critérios de inclusão e exclusão escolhidos.

Os critérios de inclusão foram os trabalhos completos com disponibilidade na íntegra, escritos em português ou inglês e com limite de temporalidade entre operíodo de 2015 a 2020. Em contrapartida os critérios de exclusão foram os estudos que não se referiam estritamente ao tema, os quais não estivessem ligados ao tema principal abordado e artigos duplicados.

Após a seleção para a síntese deste trabalho, foi realizado download de todos os artigos escolhidos e prosseguido com a leitura completa dos mesmos na íntegra para a revisão de literatura. Dessa forma, contribuindo para o processo de síntese dos resultados de vários estudos, criando um corpo de literatura compreensível.

\section{Resultados}

Foram encontrados na busca online das bases de dados 78 artigos referentes aos descritores inseridos. Dentre eles foram 38 do Pubmed, 29 do BVS, 8 do Lilacse 3 do Scielo. Após excluir os artigos repetidos foi obtido um total de 52 artigos. Em seguida, foi realizada a leitura do título e resumo destes sendo selecionados de acordo com os critérios de inclusão e exclusão, ficando 22 artigos para a incorporação deste estudo.

Na Figura 1 pode ser observada a quantidade de artigos encontrados na seleção de acordo com a natureza do estudo.

Figura 1. Figura com a frequência absoluta de artigos encontradas na busca deartigos de acordo com a natureza do estudo.

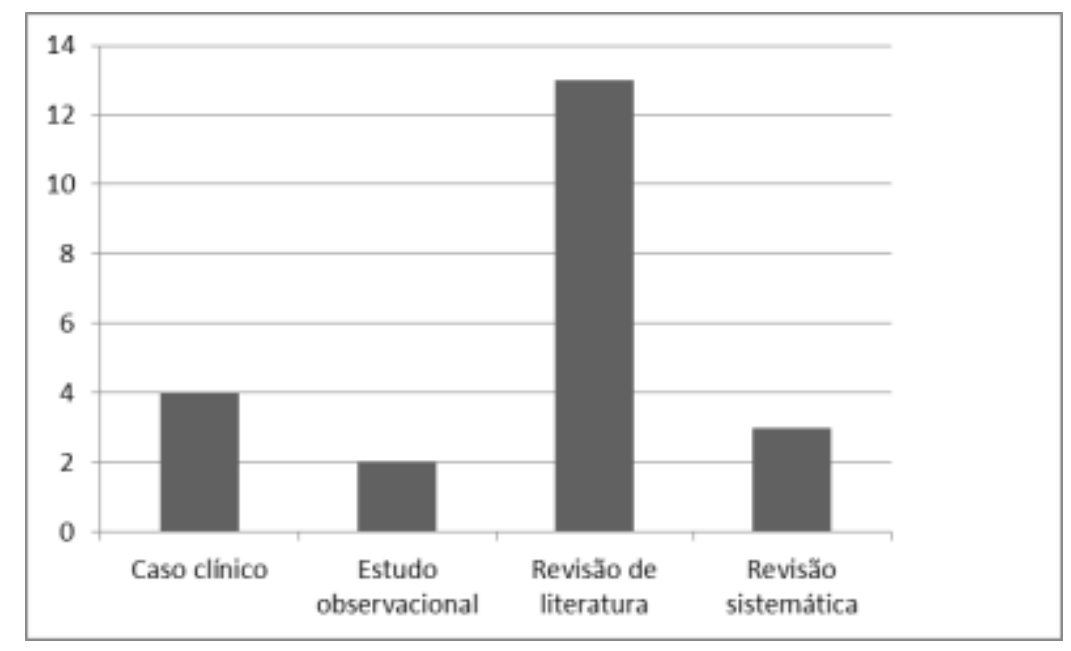

Fonte: Autores (2021). 
Como pode ser observado na Figura 1, dentre os 22 artigos selecionados foram: 13 casos clínicos, 2 estudos observacionais, 4 revisões de literatura e 3 revisões sistemáticas.

$\mathrm{Na}$ seleção final dos artigos, o maior gênero encontrado foi relatos de caso clínicos, uma vez que apresenta tanto relatos da correção com apenas o uso da toxina botulínica tipo A e o mesmo coadjuvante da gengivoplastia, gengivectomia ou cirurgia ressectiva. Estas informações de forma quantitativa podem ser observadas, por meio de frequência absoluta na Figura 2 .

Figura 2. Figura com frequência absoluta de artigos encontrada na busca de artigosde acordo com a técnica utilizada.

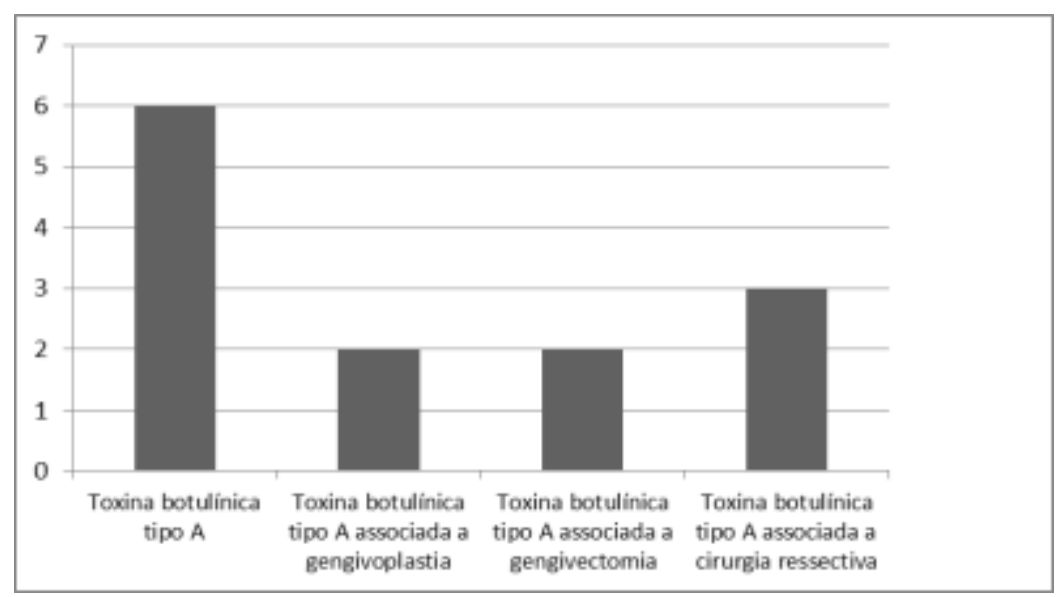

Fonte: Autores (2021).

De acordo com a Figura 2, pode ser observado que dentre os 22 artigos selecionados foram: 6 abordando a toxina botulínica de forma independente, 2 a toxina botulínica tipo A associada a gengivoplastia, 2 a toxina botulínica tipo A associada a gengivectomia e 3 a toxina botulínica tipo A associada a cirurgia ressectiva.

De maneira geral, a técnica escolhida para a correção do sorriso gengival é dada através da etiologia do paciente, podendo realizar o procedimento apenas com a toxina botulínica tipo A ou associada a outras técnicas, o que pode ser observado a partir da presente revisão de literatura.

\section{Revisão de Literatura}

\section{Sorriso Gengival}

O sorriso é uma das expressões faciais mais importantes do rosto e que está interligado com a autoestima dos pacientes (Gupta \& Kohli, 2019). Apesar de não existir um padrão absoluto de beleza, o excesso de exposição gengival pode prejudicar a aparência de acordo com os padrões de simetria facial (Mostafa, 2018). Porém, é necessário levar em consideração o conceito de beleza de cada indivíduo que é influenciado por fatores psicossocial e econômico, os quais devem ser respeitados e analisados tanto a opinião quanta a expectativa de cada paciente (Al-Fouzan et al., 2017).

O sorriso considerado estético tem como característica a relação harmônica entre estrutura dos lábios, contorno dentogengival, cor dos elementos dentários e simetria facial. Com isso, a importância do cirurgião dentista de dominar a morfologia, tonalidade e proporcionalidade anatômica, além de respeitar osprincípios biológicos, mecânicos e estéticos. Dessa forma, denomina-se sorriso gengival a exposição de mais de $2 \mathrm{~mm}$ dos tecidos periodontais ao sorrir (Moreira, Possidônio, Souza, Kinoshita \& Silveira, 2019). Entretanto, alguns autores consideram sorriso gengival apenas acima de $3 \mathrm{~mm}$ (Rego; Santos \& Pedron, 2015).

Dessa maneira o sorriso gengival pode gradualmente com a idade diminuir como consequência do surgimento de lábios caídos superior ou inferior, a diminuição do comprimento vertical, assim como desgastes dentários nos incisivos 
(Gupta \& Kohli, 2019). Por isso, é necessário que o cirurgião dentista esteja ciente dos mecanismos de compensação secundária que podem ocorrer ao tentar diminuir ou paralisar atividade de um músculo ou grupo muscular envolvidos, tendo que fazer um bom planejamento e tratar os pacientes de forma individualizada (Chagas et al., 2018).

O sorriso gengival é uma queixa bastante comum entre os pacientes no consultório, despertando o interesse dos cirurgiões dentistas em buscar e se aperfeiçoar em procedimentos temporários ou permanentes para oferecer a melhor alternativa ao paciente frente o tipo de etiologia da exibição gengival excessiva(Araújo, Cruz, Oliveira \& Canto, 2018). Nesta visão e com o crescimento da harmonização orofacial, a toxina botulínica tipo A vem liderando como formade correção precisa, podendo ser usada como procedimento independente, como pode complementar outras técnicas invasivas ou como uma escolha temporáriaenquanto se espera por uma solução permanente (Brito, Alves, Sousa, Rodrigues \& Souza, 2016).

$\mathrm{Na}$ literatura é mais relatado o uso da toxina botulínica tipo A como técnica independente associado à etiologia da hiperatividade dos músculos envolvidos no sorriso gengival e em relação ao seu uso como coadjuvante é mais descrita associada com a gengivoplastia, gengivectomia e cirurgia ressectiva, tendo excelentes resultados. (Nunes, Ferrão Junior, Teixeira, Leandro \& Guevara, 2015).

\section{Etiologia e diagnóstico}

Para descobrir a etiologia se faz necessário um diagnóstico efetivo, avaliando o sorriso gengivtravés de alguns critérios que influência diretamente na escolha do tratamento, que são à distância interlabial em repouso, a exposição dos incisivos superiores, o arco do sorriso e a proporção da largura e do comprimento dos incisivos superiores (Delpachitra, Sklavos \& Dastaran, 2018). Alguns autores sugerem fazer registros de fotografias antes e depois da aplicação da técnica (Nasr, Jabbour, Sidaoui, Haber \& Kechichian, 2015).

As três etiologias mais descritas na literatura para o sorriso gengival são erupção dentária passiva retardada, hiperatividade muscular e o osso em caso de excesso de maxila vertical. Em vista disso cada causa possui uma abordagem terapêutica diferente. Porém, as intervenções cirúrgicas são irreversíveis e invasivas, tendo a correção com toxina botulínica como reversível e não invasiva (Duruel, Ataman- Duruel, Berker \& Tozum, 2019).

O excesso de exposição da gengiva durante o sorriso pela hiperatividade muscular envolve todos os músculos funcionalmente como o lábio levantador superior, asa do nariz, elevação do canto dos lábios, o zigomático maior e menor, e o depressor nasal, os quais desempenham um papel importante no sorriso. Além disso, é apresentada na literatura como a etiologia com maior indicação para o uso da toxina botulínica tipo A (Miller \& Clarkson, 2016).

\section{Alternativas para a correção do sorriso gengival}

A literatura relata vários procedimentos como alternativas para a correção do sorriso gengival dentre elas as terapias cirúrgicas como gengivoplastia, cirurgia ortognática e ressecções ósseas. Entretanto, são procedimentos cirúrgicosconsiderados como invasivos, financeiramente caros e demorados, podendo também causar efeitos indesejáveis como cicatrizes. Para tanto, o uso da toxina botulínica é considerado por todos os autores o mais seguro, menos invasivo e com resultados positivos (Moreira, Possidônio, Souza, Kinoshita \& Silveira, 2019).

A erupção passiva retardada tem sido tradicionalmente tratada por cirurgia de alongamento de coroa envolvendo gengivectomia, gengivoplastia ou retalhos apicalmente posicionados com ou sem ressecção óssea. A cirurgia le Fort I com impactação é a abordagem padrão para correção do excesso ósseo vertical. A hiperatividade dos músculos do lábio superior tem sido tratada com ressecção da mucosa vestibular, miectomia com ressecção parcial dos músculos elevadores, dissecção subperiosteal da musculatura de elevação labial e toxina botulínica tipo A (Qian, Zhang, Hou \& Cao, 2016). 


\section{Toxina botulínica}

A toxina botulínica é uma proteína produzida pela fermentação da Clostridium botulinum, uma bactéria anaeróbica e gram-positiva, a qual é extraída sete sorotipos(A, B, C, D, E, F e G) (Pedron, 2018). Entretanto, de acordo todos os autores atoxina tipo A é a mais utilizada por ser mais efetiva e apresentar menor custo, sendoum tratamento seguro, podendo substituir procedimentos invasivos como cirúrgico etendo bons resultados (Chen, Oranges, Giordano, Huang \& Wang, 2019).

Esta toxina vem sendo estudada desde o final dos anos de 1970 para diversos fins de tratamento, inclusive associados à contração muscular excessiva. Com isso vem ganhando espaço na medicina estética, sendo aprovada pela ANVISA no Brasil em 2000, pela sua importância devido às suas propriedades terapêuticas. Entretanto, apesar de correção do sorriso gengival com esta técnica ser bastante frequente no dia a dia clínico, na literatura este assunto ainda é escasso incluindo questões fundamentais como seu diagnóstico e tratamento (Moura, Vasconcelos Neto, Correa Neto, Vasconcelos \& Moura, 2017).

É recomendado que a comercialização da toxina seja feito na forma a vácuo, por ser bastante segura. O uso deve ser feito com menor tempo possível e evitar armazenar o produto já pronto (Mostafa, 2018). Porém, devido ao vácuo, deve-seter cuidado no momento da diluição na solução salina para que não aconteça de forma rápida resultando em quebra das moléculas e em relação à recongelar podeter perca da eficácia da toxina ao longo do tempo (Nunes, Ferrão Junior, Teixeira, Leandro \& Guevara, 2015).

O mecanismo de ação da toxina botulínica tipo A atua bloqueando um neurotransmissor chamado acetilcolina responsável pela contração. Consequentemente, causando um relaxamento muscular temporário (Gupta \& Kohli, 2019). Entretando, em relação à duração do enfraquecimento do músculo foi notado nos relatos de casos clínicos que varia de acordo com o metabolismo e a hiperatividade dos músculos envolvidos no sorriso, tendo duração entre 3 a 6 meses (Farias Neto, Santos, Galvão, Lemos \& Vilanova, 2016).

As contraindicações do uso da toxina botulínica são para indivíduos com hipersensibilidade à própria toxina, gestantes, lactantes, portadores de doenças musculares e neurodegenerativas como miastenia gravis e doença de Charcot; e o uso simultâneo de antibiótico amino glicosídico, que pode resultar na potencializaçãoda ação da toxina (Bonin, Silva, Shimizu \& Schimizu, 2019).

\section{Aplicação}

A aplicação da toxina botulínica tipo A deve-se inicialmente realizar as marcações dos pontos de punção no paciente. Durante a aplicação, os músculos precisam estar em repouso e em relação à dosagem para pacientes com 3 a $5 \mathrm{~cm}$ é recomendada entre $2 \mathrm{U}$ a $3 \mathrm{U}$ por ponto. A agulha é de $4 \mathrm{~mm}$ e a injeção tem que ser numa direção oblíqua à superfície da pele de forma delicada com pressão levemente sobre o êmbolo (Araújo, Cruz, Oliveira \& Canto, 2018). Mas, a localização do ponto e a dosagem vão depender do tamanho da exposição do sorriso gengival (Gupta \& Kohli, 2019).

Dependendo do paciente pode ter assimetria labial, tendo que durante aaplicação da toxina para a correção do sorriso gengival ser feita com doses diferentes em ambos os lados. Porém, não existem relatos entre os autores sobre diferença de resultados em comparação com as doses iguais (Duruel, 2019). Na literatura alguns autores descrevem o "Ponto Yonsei" como técnica de aplicação, a qual é definida como uma única injeção que atingi bilateralmente os músculos levantadores do lábio superior e os músculos zigomáticos. A localização da injeção no "Ponto Yonsei" é a $1 \mathrm{~cm}$ lateral à ala horizontalmente e $3 \mathrm{~cm}$ acima da linha dos lábios, verticalmente (Miller \& Clarkson, 2016).

O resultado da terapia com a toxina botulínica tipo A na correção do sorriso gengival após a aplicação diminui gradualmente com o tempo, embora ainda mantida satisfatoriamente e não tendo retornado a exposição inicial nas primeiras 12 semanas (Chen, Oranges, Giordano, Huang \& Wang, 2019). 
Alguns autores relataram um número significativo em relação a pacientes que apresentaram um aumento dos intervalos de reaplicação da toxina, isso se dar pela regularidade da repetição da terapia fazendo com que haja à diminuição da força muscular que ocorre após várias aplicações de toxina botulínica tipo A (Duruel, 2019). Todavia, é preciso reavaliar o paciente a cada retorno entre 4 a 6 meses para ajustar a dosagem e as aplicações (Nasr, Jabbour, Sidaoui, Haber \& Kechichian, 2015).

\section{Pós-operatório}

O pós-operatório cabe ao cirurgião dentista fazer o acompanhamento e solicitar retorno à clínica após 15 dias para monitorar os resultados. Vale ressaltar que o paciente deve ser informado que os resultados com uso da toxina botulínica não é imediato e que é temporário, precisando retornar após 4 a 6 meses para uma nova aplicação (Qian, Zhang, Hou \& Cao, 2016). Além do mais deve ser feita recomendações como não fazer uso de antiinflamátorios esteroides, evitar fazer compressões na região, assim como viagens aéreas nos primeiros dias e possíveis efeitos colaterais com dor de cabeça (Bonin, Silva, Shimizu \& Schimizu, 2019).

\section{Vantagens e desvantagens}

De acordo com os autores, a maior vantagem da correção do sorriso gengival com as técnicas tradicionais é não ser um tratamento invasivo, sendo um procedimento reversível, quase sempre indolor e de custo acessível (Pedron \& Mangano, 2018). Entretanto, alguns autores discordam em relação ao custo acessível, devido a necessidade de ter que fazer novas aplicações a cada 4 ou 6 meses, devido a retomada da contração dos músculos envolvidos (Chen, Oranges, Giordano, Huang \& Wang, 2019).

As desvantagens da técnica é que o efeito não é imediato, custando alguns dias para que os efeitos clínicos apareçam, pois geralmente ocorre entre 2 a 10 dias após o procedimento. Além disso, tem duração limitada por aproximadamente 3 a 6 meses, dependendo do organismo, necessitando de uma nova aplicação. Por isso, é necessário o acompanhamento do profissional e a solicitação da retomada do paciente a clínica com 7 ou 15 dias após a aplicação para a avaliação dos resultados (Al-Fouzan et al., 2017).

A aplicação da toxina botulínica pode estar associado a eventos adversos como dor no local da injeção, hematoma, infecção, edema, disfonia, disfagia, ptose ou alongamento do lábio superior e assimetria do sorriso. Em vista disso, é importante que o cirurgião dentista tenha domínio da técnica e localização da punção, assim como respeite a dosagem de acordo com a hiperexposição dos tecidos periodontais (Miller \& Clarkson, 2016).

\section{Discussão}

O sorriso gengival, conceitualmente, caracteriza-se por uma quantidade excessiva de gengiva visível enquanto o indivíduo sorri (Farias Neto, Santos, Galvão, Lemos \& Vilanova, 2016). Diante dessa consideração, vale ressaltar que existe um duelo na literatura, pois alguns autores consideram sorriso gengival a partir de $3 \mathrm{~mm}$, enquanto outros já consideram com $2 \mathrm{~mm}$.

Dessa maneira, a percepção de cirurgiões-dentistas e leigos em odontologia pode divergir em relação ao conceito de estética, resultando em dificuldade em propor que essas alterações estéticas devam ou não ser corrigidas, como no caso o sorriso gengival (Al-Fouzan et al., 2017). Porém, a opinião e as expectativas do paciente devem ser sempre respeitadas e analisadas. 
A etiologia do excesso da exposição da gengiva durante o sorriso é bastante variada e o tratamento pode ser mais conservador ou mais invasivo dependendo da causa e da escolha (Nunes, Ferrão Junior, Teixeira, Leandro \& Guevara, 2015).

Os procedimentos consistem em gengivoplastia, gengivectomia, reposicionamento labial, cirurgia ortognática, tratamento ortodôntico ou aplicação de toxina botulínica tipo A (Chen, Oranges, Giordano, Huang \& Wang, 2019). Todavia, o uso da toxina botulínica pode ser usada isoladamente ou como coadjuvante de outra técnica com resultados bastante satisfatórios, como foi possível ser observado a partir dos estudos encontrados na presente revisão, como por exemplo, a associação desta técnica com as cirurgias ressectivas.

A escolha e o grande uso da toxina botulínica tipo A, é devido ao fato de ser mais eficiente, possuir um menor custo e apresentar resultado temporário entre 3 a 6 meses, variando entre cada paciente (Moreira, Possidônio, Souza, Kinoshita \& Silveira, 2019).

Além disso, todos os autores citam a toxina botulínica como procedimento de primeira linha, devido a segurança e a facilidade de aplicação, uso de doses reduzidas de ação rápida, baixo risco, efeito reversível e eficaz. Tendo seu efeito relacionado à localização da aplicação e dose utilizada que trará resultados positivos ou negativos (Duruel, Ataman-Duruel, Berker \& Tozum, 2019). Entretanto, apesar dessa alternativa de correção do sorriso gengival ser bastante segura necessita ser aplicada por um profissional especializado e de forma cautelosa para evitar complicações (Chen, Oranges, Giordano, Huang \& Wang, 2019).

Em relação à aplicação, a quantidade do produto a ser aplicado deve ser dividido igualmente para ambos os lados e aplicado por meio de uma agulha de $4 \mathrm{~mm}$, a dosagem vai depender da exposição gengival do paciente (Chagas et al., 2018). Porém, a individualização do protocolo deve ser respeitada uma vez que pacientes podem possuir um sorriso assimétrico.

Embora a resistência ao sorotipo A da toxina botulínica seja rara, a literatura relata que a mesma pode produzir anticorpos que inibem os efeitos da toxina. Isso pode acontecer devido a uma alta dose durante um curto período de tempo, aumentando o risco de desenvolver anticorpos neutralizantes contra o produto aplicado. Além disso, pacientes podem substituir por outra forma de toxina se forem imunorresistentes ao sorotipo A (Brito, Alves, Sousa, Rodrigues \& Souza, 2016). Com base disso, vale ressaltar a limitação de estudos clínicos sobre a substituição do sorotipo, em relação à dosagem, vantagens e desvantagens ao considerar os casos clínicosselecionados.

Alguns autores relataram como forma de prevenção, contra complicações à aplicação da toxina botulínica, a utilização em duas sessões, preferindo injetar doses baixas inicialmente, e em seguida retocar se necessário. Com isso evitando toxicidade da toxina e danos ao nervo ou músculo (Moura, Vasconcelos Neto, Correia Neto, Vasconcelos \& Moura, 2017). Além disso, os autores citam que a aplicação da toxina em doses baixas ajuda ao organismo a não criar tolerância à aplicação da mesma. (Rego, Santos \& Pedron, 2015).

Vale destacar que o cirurgião dentista é autorizado a fazer o uso da toxina botulínica, preenchedores faciais na região orofacial e em estruturas anexas e afins na Resolução 198/2019 pelo Conselho Federal de Odontologia (CFO) (Chen, Oranges, Giordano, Huang \& Wang, 2019).

Apesar da resistência enfrentada pelos dentistas em relação a realizar o procedimento, estes profissionais são bem versados na anatomia e fisiologia da musculatura do rosto e sua contribuição para o sorriso, possuindo então uma base sólida. Porém, se faz necessário um curso de especialização para compreender as aplicações clinica e adequadas da toxina botulínica na região orofacial para um diagnóstico preciso e resultados bastante satisfatórios na correção do sorriso gengival com essa alternativa.

A presente revisão buscou avaliar os estudos em relação ao uso da toxina botulínica na correção do sorriso gengival e seu uso pelo cirurgião-dentista. Apesar da ampla aceitação de sua eficácia e segurança, ainda é necessário que haja mais 
evidências científicas, como ensaios clínicos randomizados bem delineados com um mínimo de 6 meses de acompanhamento e revisões sistemáticas para fortalecer o embasamento científico, pois é uma técnica reversível e com resultados estéticos positivos, sendo a melhor opção para quem deseja realizar a correção do sorriso gengival de forma não invasiva.

\section{Conclusão}

Diante da revisão de literatura foi possível reconhecer que o uso da toxina botulínica tipo A na correção do sorriso gengival apresenta-se com alternativa segura, eficácia e com resultados bastante satisfatórios tanto quando aplicada de forma independente ou associada a outras técnicas cirúrgicas. Além disso, para o alcance desse sucesso se faz necessário que a aplicação seja feito por um profissional que realize um correto diagnóstico e seja capacitado.

\section{Referências}

Araújo J. P., Cruz J., Oliveira J. X. \& Canto A. M. (2018). Botulinum Toxin Type-A as an alternative treatment for gummy smile: a case report. Dermatol Online J, 24 (7), 37-38.

Al-fouzan A. F., Mokeem L. S., Al-Saqat R. T., Alfalah M., Alharbi M. \& Al-Samary A. (2017). Toxina botulínica para o tratamento do sorriso gengival. The Journal Of Contemporary Dental Practice, 6 (18),474-478.

Bonin F. A., Silva R. D. da, Shimizu R. H. \& Schimizu I. A.(2019). Avaliação da percepção e da satisfação estética de pacientes que foram tratados com toxina botulínica tipo A para correção do sorrisogengival. Full Dent. Sci, 10 (39), 150- 154.

Brito E. C. D. de, Alves W. de A., Sousa J. N. L. de, Rodrigues R. de Q. F. \& Souza M. M. L. de (2016). Gengivectomia: gengiplastia associada à toxina botulínica paracorreção de sorriso gengival. Revista Sobrape, 26 (3), 50-56.

Chagas T. F., Almeida N. V. de, Lisboa C. O., Ferreira D. M. T. P., Mattos C. T. \& Mucha J. N (2018). Duration of effectiveness of Botulinum toxin type A in excessive gingival display: a systematic review and meta-analysis. Brazilian OralResearch, 32 (2), 327-330. 10.1590/1807-3107bor-2018.vol32.0030

Chen G., Oranges C. M., Giordano S., Huang R. \& Wang W. (2019). Horizontal animation deformity as unusual complication of neurotoxin modulation of the gummysmile. Dermatol Online J, 25 (8), 34-37.

Delpachitra S. N., Sklavos A. W. \& Dastaran M. (2018). Clinical uses of botulinumtoxin A in smile aesthetic modification. British Dental Journal, 225 (6), 502-506. doi: 10.1038/sj.bdj.2018.755.

Duruel O., Ataman-Duruel E. T., Berker E. \& Tözüm T. F. (2019). Treatment of Various Types of Gummy Smile With Botulinum Toxin-A. Journal Of Craniofacial Surgery, 30 (3), 876-878. doi: 10.1097/SCS.0000000000005298.

Duruel O. (2019). Ideal Dose and Injection Site for Gummy Smile Treatment withBotulinum Toxin-A: a systematic review and introduction of a case study. The International Journal Of Periodontics \& Restorative Dentistry, 39 (4),167-173. 10.11607/prd.3580.

Farias Neto A., Santos G. C. de O., Galvão L. T., Lemos J. C. \& Vilanova L. S. R. (2016). Aplicação da toxina botulínica tipo A na correção do sorriso gengival. Full Dentistry In Science, 29 (8), 55-57.

Gupta N. \& Kohli S. (2009). Evaluation of a neurotoxin as an adjunctive treatment modality for the management of gummy smile. Indian DermatologyOnline Journal, 10 (5), 560-664. doi: 10.4103/idoj.IDOJ_365_18

Miller J. \& Clarkson E. (2016). Botulinum Toxin Type A. Dental Clinics Of NorthAmerica, 60 (2), 509-521. d10.1016/j.cden.2015.11.007.

Moura L. A., Vasconcelos Neto R, de S., Correa Neto A. de O., Vasconcelos L. K. M. F. \& Moura R. R. (2017). Associação de técnica cirúrgica e toxina botulínica para correção desorriso gengival/ Association of surgical technique and botulinum toxin in gummy smile management case report. Implantnewsperio, 2 (3) 515-520.

Moreira D. C., Possidônio F. S., Souza F. S. de, Kinoshita A. M. O. \& Silveira E. M. V. (2019). Application of botulinum toxin type A in gummy smile: case report. Rgo - Revista Gaúcha de Odontologia, 67, 34-37. 10.1590/1981-86372019000133555

Mostafa D. (2018). A successful management of sever gummy smile using gingivectomy and botulinum toxin injection: a case report. International Journal OfSurgery Case Reports, 42 (1), 169-174. doi: 10.1016/j.ijscr.2017.11.055

Nasr M. W, Marwan W., Jabbour S. F., Sidaoui J. A., Haber, R. N. \& Kechichian E. G. (2015). Botulinum Toxin for the Treatment of Excessive Gingival Display: a systematic review. Aesthetic Surgery Journal, 36 (1), 82-88. doi: 10.1093/asj/sjv082

Nunes L., Ferrão Junior J. P., Teixeira S. A. F., Leandro L. F. L. \& Guevara H. A. G. (2015). Tratamiento de lasonrisa gingival con la toxina botulínica tipo A: caso clínico. Revista Española de Cirugía Oral y Maxilofacial, 37 (4) 229-232. 10.1016/j.maxilo.2014.03.001

Pedron I. G. \& Aulestia-viera P. V. (2016). La toxina botulínica como adyuvante en el tratamiento de la sonrisa gingival. Revista Clínicade Periodoncia, Implantología y Rehabilitación Oral, 10 (2), 24-27. 10.1016/j.piro.2016.06.001. 
Research, Society and Development, v. 10, n. 9, e21810917930, 2021

(CC BY 4.0) | ISSN 2525-3409 | DOI: http://dx.doi.org/10.33448/rsd-v10i9.17930

Pedron I. G. \& Mangano A. (2018). Gummy Smile Correction Using Botulinum Toxin With Respective Gingival Surgery. Journal Of Dentistry,3 (19), 248252. https://www.ncbi.nlm.nih.gov/pmc/articles/PMC6092459/

Pedron I. G. (2018) Type A Botulinum Toxin as Complement to Gingivoplasty in the Treatment of Gummy Smile. Case Report. UniversitasOdontologica, 37 (78), 33-37, 10.11144/Javeriana.uo37-78.tabt

Qian W., Zhang Y, Lv W., Hou Y., Cao Q. \& Fan J (2016). Application of Local Injection of Botulinum Toxin A in Cosmetic Patients with Congenital Drooping Mouth Corner. Aesthetic Plastic Surgery, 40 (6), 926-930. 10.1007/s00266-016-0711-0

Rego R. V., Santos J. E. \& Pedron I. G. (2015). Complementação da cirurgia gengival ressectiva pela aplicação da toxina botulínicano manejo do sorriso gengival em paciente ortodôntico. Orthodontics Science And Practice, 8 (29), 74-78. 